\title{
The evolution of bacterial LuxI and LuxR quorum sensing regulators
}

\author{
Kendall M. Gray† and James R. Garey \\ Author for correspondence: Kendall M. Gray. Tel: +1 206543 9396. Fax: +1 2066166749. \\ e-mail:kmg@u.washington.edu
}

Department of Biology, University of South Florida, 4202 E. Fowler Ave, SCA 110, Tampa, FL 33620, USA

\begin{abstract}
Quorum sensing is a widespread form of bacterial communication in which individual cells produce and respond to specific $\boldsymbol{N}$-acyl homoserine lactone signal metabolites. The different autoinducer synthases that generate these signals and the receptor/activator proteins that mediate the cell's response to them constitute evolutionarily conserved families of regulatory proteins known as the Luxl and LuxR families, respectively. We have performed a phylogenetic analysis of $\mathbf{7 6}$ individual LuxI and LuxR homologues present in diverse members of the Gram-negative Proteobacteria. The results were consistent with an early origin for these regulators during the evolution of the Proteobacteria, with functional pairs of luxl and luxR genes possibly coevolving as regulatory cassettes. In many cases, specific Luxl and LuxR family members appeared to have been inherited horizontally. In particular, those species containing multiple Luxl and/or LuxR homologues usually appeared to have obtained each individual homologue or functional pair of homologues from an independent source. Because multiple homologues interact to form regulatory cascades, this finding suggests that hierarchical signalling pathways can potentially evolve by the sequential integration of pre-existing regulatory circuits acquired from diverse sources.
\end{abstract}

Keywords: intercellular signalling, cell-cell communication, horizontal gene transfer

\section{INTRODUCTION}

Intercellular communication plays a central role in the physiology and development of living organisms. Even unicellular organisms such as bacteria will frequently coordinate their activities so that populations of cells can function as multicellular entities (Shapiro, 1998). Such coordination necessarily requires cell-cell communication, often by means of the production and release of specific signal compounds by individual cells. The extracellular accumulation of these self-produced signals denotes the presence of a relatively dense population of cells and thus the appropriateness of coordinated group behaviour. Bacterial cell-cell communication of this sort is known as quorum sensing.

The best-studied mechanisms of quorum sensing are found among the Gram-negative Proteobacteria, which use $\mathrm{N}$-acyl homoserine lactone 'autoinducers' as signal

† Present address: Department of Microbiology, Box 357242, University of Washington, Seattle, WA 98195-7242, USA. molecules (Greenberg, 2000; Parsek \& Greenberg, 2000; Swift et al., 1999). With few exceptions, the proteins that synthesize these autoinducers constitute an evolutionarily conserved family of homologues known as the LuxI family of autoinducer synthases. The proteins that act as the specific response elements for autoinducers form another evolutionarily conserved group known as the LuxR family of transcriptional regulators.

LuxI and LuxR homologues have been identified in diverse species throughout the Gram-negative Proteobacteria, including phototrophic purple non-sulfur bacteria, marine vibrios, rhizosphere bacteria, enteric commensals and opportunistic pathogens of plants and animals. The LuxI and LuxR homologues present in these species regulate such disparate functions as exoenzyme synthesis, conjugation, antibiotic production, luminescence and biofilm formation (Greenberg, 2000; Parsek \& Greenberg, 2000; Swift et al., 1999).

The genetic organization of LuxI and LuxR family members is as diverse as their function. Homologues of luxI and $\operatorname{luxR}$ genes have been identified both on bacterial chromosomes and on extrachromosomal elements such as the symbiosis (Sym) and tumour-inducing 
Table 1. Quorum sensing homologues included in this study

\begin{tabular}{|c|c|c|c|c|}
\hline Organism & $\begin{array}{c}\text { LuxI } \\
\text { homologue* }\end{array}$ & $\begin{array}{c}\text { LuxR } \\
\text { homologue* }\end{array}$ & $\begin{array}{l}\text { Orientation of } \\
\text { adjacent genes }\end{array}$ & $\begin{array}{l}\text { GenBank accession } \\
\text { no. or reference }\end{array}$ \\
\hline \multicolumn{5}{|l|}{$\alpha$-Proteobacteria } \\
\hline Agrobacterium tumefaciens (pTiA6; pTiR10) (oct) $†$ & TraI & TraR & Non-adjacent & L17024; L08596 \\
\hline Agrobacterium tumefaciens (pTiR10) (oct) $\dagger$ & - & $\operatorname{TrlR} \ddagger$ & Non-adjacent & U60011 \\
\hline Agrobacterium tumefaciens (pTiC58) (nop) $\dagger$ & TraI & TraR & Non-adjacent & L22207; Z15003 \\
\hline Agrobacterium tumefaciens (pTi-SAKURA) (nop) $\dagger$ & TraI & - & Non-adjacent & $\mathrm{AB} 006858$ \\
\hline Agrobacterium vitis $(\mathrm{pTi} 2608)(\mathrm{o} / \mathrm{c}) \dagger$ & - & TraR & Non-adjacent & Z22732 \\
\hline Rhizobium etli & RaiI & RaiR & $\rightarrow \rightarrow$ & U92712; U92713 \\
\hline Rhizobium leguminosarum $\mathbb{S}$ & CinI & CinR & $\rightarrow \rightarrow$ & AF210630 \\
\hline Rhizobium leguminosarum (pRL1JI) $\mathbb{S}$ & RhiI & RhiR & Non-adjacent & $\begin{array}{l}\text { Rodelas et al. (1999); } \\
\text { M98835 }\end{array}$ \\
\hline Rhizobium leguminosarum (pRL1JI)S & - & BisR & Non-adjacent & $\begin{array}{l}\text { J. A. Downie (unpublished } \\
\text { results) }\end{array}$ \\
\hline Rhizobium leguminosarum (pRL1JI) & - & TriR & Non-adjacent & $\begin{array}{l}\text { J. A. Downie (unpublished } \\
\text { results) }\end{array}$ \\
\hline Rhizobium sp. NGR 234 (pSym) $\mathbb{S}$ & TraI & TraR & Non-adjacent & AE000068; AE000069 \\
\hline Rhizobium sp. NGR 234 (pSym)S & - & $\mathrm{Y} 4 \mathrm{qH}$ & Non-adjacent & AE000093 \\
\hline Rhodobacter sphaeroides & CerI & CerR & $\rightarrow \rightarrow$ & AF016298 \\
\hline \multicolumn{5}{|l|}{$\beta$-Proteobacteria } \\
\hline Burkbolderia cepacia & CepI & CepR & $\leftarrow \rightarrow$ & AF019654 \\
\hline Chromobacterium violaceum & CviI & CviR & $\rightarrow \leftarrow$ & $\begin{array}{l}\text { M. K. Winson (unpublished } \\
\text { results) }\end{array}$ \\
\hline Ralstonia solanacearum & SolI & SolR & $\leftarrow \rightarrow$ & AF02184 \\
\hline \multicolumn{5}{|l|}{$\gamma$-Proteobacteria, aeromonads } \\
\hline Aeromonas bydrophila & AhyI & AhyR & $\leftarrow \rightarrow$ & X89469 \\
\hline Aeromonas salmonicida & AsaI & AsaR & $\leftarrow \rightarrow$ & U65741 \\
\hline \multicolumn{5}{|l|}{$\gamma$-Proteobacteria, enterobacteria } \\
\hline Pantoea (Enterobacter) agglomerans & EagI & $\operatorname{EagR}$ & $\rightarrow \leftarrow$ & X74300 \\
\hline Erwinia carotovora subsp. carotovora SCC 3193 & ExpI & ExpR & $\rightarrow \leftarrow$ & $\mathrm{X} 72891 ; \mathrm{X} 80475$ \\
\hline Erwinia carotovora subsp. carotovora 71 & HslI (ExpI) & HslR (ExpR) & $\rightarrow \leftarrow$ & L40174 \\
\hline Erwinia carotovora subsp. carotovora GS101 & CarI (ExpI) & $\operatorname{RexR}(\operatorname{ExpR})$ & $\rightarrow \leftarrow$ & X74299 \\
\hline Erwinia carotovora subsp. carotovora GS101 & - & CarR & Non-adjacent & U17224 \\
\hline Erwinia carotovora subsp. carotovora SCRI 193 & - & CarR & Non-adjacent & Holden et al. (1998) \\
\hline Erwinia carotovora subsp. betavasculorum & EcbI & EcbR & $\rightarrow \leftarrow$ & AF001050 \\
\hline Erwinia chrysanthemi NCPPB 1066 & EchI (ExpI) & $\operatorname{EchR}(\operatorname{ExpR})$ & $\rightarrow \leftarrow$ & $\mathrm{U} 45854$ \\
\hline Escherichia coli & - & SdiA & Non-adjacent & AE000284 \\
\hline Pantoea (Erwinia) stewartii & EsaI & EsaR & $\rightarrow \leftarrow$ & L32184 \\
\hline Erwinia chrysanthemi 3937 & ExpI & ExpR & $\rightarrow \leftarrow$ & X96440 \\
\hline Salmonella typhimurium & - & SdiA & Non-adjacent & U88651 \\
\hline Serratia liquefaciens & SwrI & SwrR & $\rightarrow \leftarrow$ & $\begin{array}{l}\text { U22823; M. Givskov \& } \\
\text { M. K. Winson } \\
\text { (unpublished results) }\end{array}$ \\
\hline Serratia marcescens & SmaI & SmaR & $\rightarrow \leftarrow$ & AJ275980 \\
\hline Serratia marcescens & - & CarR & Non-adjacent & AF012907 \\
\hline Yersinia enterocolitica & YenI & YenR & $\rightarrow \leftarrow$ & X76082 \\
\hline \multicolumn{5}{|l|}{$\gamma$-Proteobacteria, pseudomonads } \\
\hline Pseudomonas aeruginosa $\mathrm{PAO} 1$ & LasI & LasR & $\rightarrow \rightarrow$ & D90832; L04681 \\
\hline Pseudomonas aeruginosa $\mathrm{PAO} 1$ & RhlI & RhlR & $\rightarrow \rightarrow$ & $\mathrm{U} 40458$ \\
\hline Pseudomonas aeruginosa PAO1 & VsmI (RhlI) & VsmR (RhlR) & $\rightarrow \rightarrow$ & U15644 \\
\hline Pseudomonas aeruginosa PG201 & RhlI & RhlR & $\rightarrow \rightarrow$ & L08962 \\
\hline Pseudomonas aureofaciens & PhzI & PhzR & $\rightarrow \leftarrow$ & L33724; L32729 \\
\hline Pseudomonas fluorescens & PhzI & PhzR & $\rightarrow \leftarrow$ & L48616 \\
\hline Pseudomonas syringae pv. tabaci & PsyI & PsyR & $\rightarrow \leftarrow$ & U39802 \\
\hline Pseudomonas syringae pv. syringae & AhlI & $A h l R$ & $\rightarrow \leftarrow$ & AF072537 \\
\hline
\end{tabular}


Evolution of quorum sensing regulators

Table 1 (cont).

\begin{tabular}{|lcccc|}
\hline Organism & $\begin{array}{c}\text { LuxI } \\
\text { homologue* }\end{array}$ & $\begin{array}{c}\text { LuxR } \\
\text { homologue* }\end{array}$ & $\begin{array}{c}\text { Orientation of } \\
\text { adjacent genes }\end{array}$ & $\begin{array}{c}\text { GenBank accession } \\
\text { no. or reference }\end{array}$ \\
\hline -Proteobacteria, vibrios & & & & U69677 \\
Vibrio anguillarum & VanI & VanR & $\rightarrow \leftarrow$ & M19039 \\
Vibrio (Photobacterium) fischeri ATCC 7744 & LuxI & LuxR & $\leftarrow \rightarrow$ & M96844 \\
Vibrio (Photobacterium) fischeri ES114 & LuxI & LuxR & $\leftarrow \rightarrow$ & Y00509 \\
Vibrio (Photobacterium) fischeri MJ1 & LuxI & LuxR & $\leftarrow \rightarrow$ & \\
\hline
\end{tabular}

* When different names are reported for what may be the same genetic locus, the alias is listed followed by the first-reported name in parentheses. Homologues written in italics were unavailable for this study.

† The tumour-inducing ( $\mathrm{Ti}$ ) plasmids of Agrobacterium spp. are grouped according to type as either octopine-producing (oct), nopalineproducing (nop) or octopine/cucumopine-producing $(\mathrm{o} / \mathrm{c}$ ). The homologues listed are present on the Ti plasmids specifically.

¥ The $t r l R$ gene contains a frameshift mutation in the $3^{\prime}$ end of the normal reading frame (Oger et al., 1998; Zhu \& Winans, 1998). This point mutation was corrected to generate a gene product that more accurately reflects its relationship to other members of the LuxR family.

\$Homologues listed for Rhizobium spp. are present on the chromosome unless a symbiosis (Sym) plasmid is designated (e.g. pRL1JI), in which case the homologues are present on the Sym plasmid itself.

(Ti) plasmids of Rhizobium and Agrobacterium spp., respectively. Many bacterial species even contain multiple LuxI and/or LuxR homologues for the production or detection of multiple, distinct signals. Multiple LuxR homologues can also permit the independent activation of different gene functions in response to a single autoinducer signal (McGowan et al., 1995; Pirhonen et al., 1993).

The question of where these two families of quorum sensing regulators first arose and how they came to be disseminated across such wide evolutionary distances has yet to be addressed. We have therefore performed a phylogenetic analysis of 76 LuxI and LuxR family members. Our results suggest that these quorum sensing regulators arose early in the evolution of the Proteobacteria and subsequently diverged within each group of organisms, although instances of horizontal gene transfer have also occurred. Surprisingly, most bacteria that contain multiple LuxI and/or LuxR family members appear to have acquired each of their different homologues from separate sources. This result provides new insights into the possible evolution of hierarchical signalling cascades in bacteria.

\section{METHODS}

DNA sequences. The LuxI and LuxR homologues considered in this analysis are listed in Table 1 . Published sequences were obtained from GenBank, while unpublished sequences were generously provided by individual colleagues. MalT of Escherichia coli (accession no. M13585), EpsR of Ralstonia solanacearum (M61197) and FixJ of Sinorhizobium meliloti (Z21854) were tested as outgroups for the analysis of LuxR family members (Henikoff et al., 1990). Prealigned 16S rRNA gene sequences were obtained from the rRNA WWW Server (Van de Peer et al., 1998).

Sequence alignment and analysis. Amino acid sequences were aligned using the default parameters of CLUSTALX (Higgins \& Sharp, 1989) and adjusted by eye using GeneDoc (Nicholas \& Nicholas, 1997). For purposes of comparison, a second set of alignments was made based on the published alignments of 17 LuxI homologues (Fuqua \& Eberhard, 1999) and 15 LuxR homologues (Stevens \& Greenberg, 1999). Sites containing gaps were excluded from the analysis to reduce systematic errors. Alignments were analysed using PAUP* version 4.0b4 (Swofford, 2000) to produce maximum-parsimony trees. Confidence in maximum-parsimony trees was determined by analysing 100 bootstrap replicates. Neighbourjoining trees were produced with the MEGA program (Kumar et al., 1994) using the $\gamma$ model of amino acid substitution with a shape parameter $a$ of $2 \cdot 0$. Confidence in neighbour-joining trees was determined by analysing 500 bootstrap replicates. All trees were drawn with nodes collapsed where bootstrap support was less than $50 \%$.

When tree topologies disagreed with the groupings suggested by rRNA analysis, alternative trees were tested statistically both by a pairwise parsimony method (Templeton, 1983) and by comparison of log-likelihood differences (Kishino \& Hasegawa, 1989), each as implemented by PAUP*. Analysis of the LuxI family tree was performed using a strict consensus of the eight most parsimonious trees derived from the modified CLUSTAL analysis. Analysis of the LuxR family tree was performed using a strict consensus of the two most parsimonious trees, while analysis of a combined LuxI/LuxR tree was performed on a strict consensus of the six most parsimonious trees. In all cases, alternative topologies were considered significantly different if $P$ was less than $0 \cdot 05$.

\section{RESULTS AND DISCUSSION}

\section{Host bacterial phylogeny as determined by rRNA analysis}

The bacteria included in this study can be grouped according to $16 \mathrm{~S}$ rRNA gene sequences into the $\alpha$ Proteobacteria (comprising the genera Agrobacterium, 


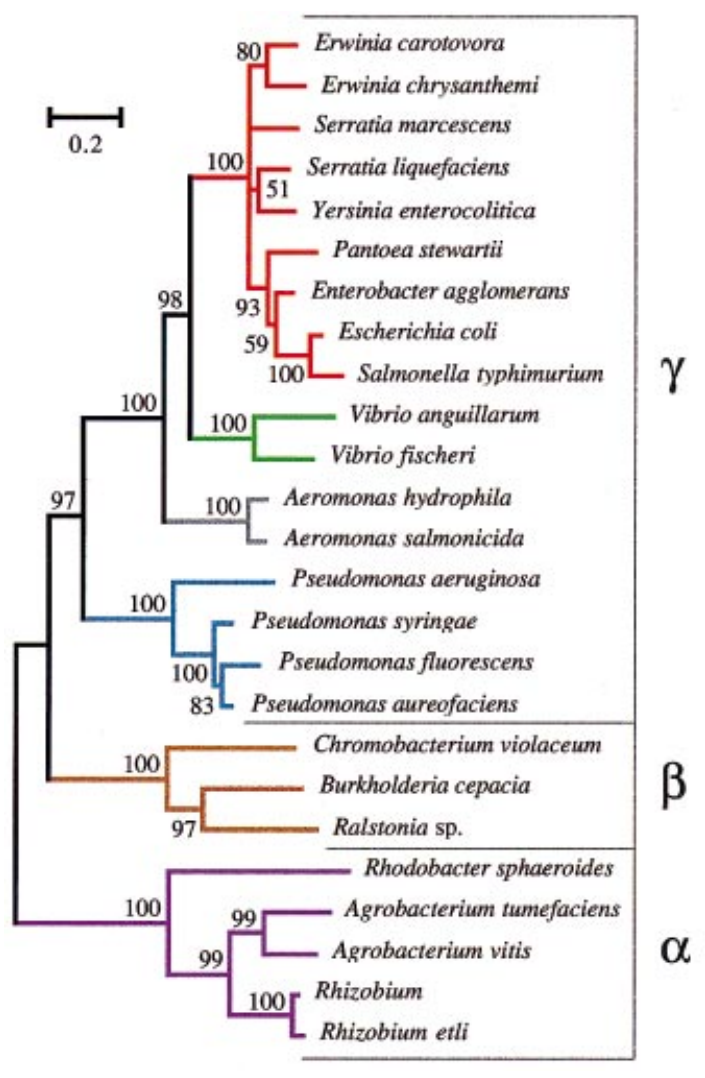

Fig. 1. Neighbour-joining tree of $16 \mathrm{~S}$ rRNA sequences from bacteria that contain Luxl and/or LuxR family members. Species belonging to the $\alpha-, \beta$ - and $\gamma$-Proteobacteria are grouped within brackets. Different colours within the $\gamma$-Proteobacteria identify pseudomonads (blue), aeromonads (grey), vibrios (green) and enterobacteria (red). Scale bar indicates the mean number of substitutions per site. Bootstrap values as the percentage of 500 replicates are shown for each branch.

Rhizobium and Rhodobacter), the $\beta$-Proteobacteria (Burkholderia, Chromobacterium and Ralstonia) and the $\gamma$-Proteobacteria (Fig. 1; Woese, 1987). The $\gamma$ Proteobacteria can be subdivided into families representing the pseudomonads (Pseudomonas), aeromonads (Aeromonas), vibrios (Vibrio) and enterobacteria (Enterobacter, Erwinia, Escherichia, Pantoea, Salmonella, Serratia and Yersinia) (Table 1, Fig. 1). These $16 \mathrm{~S}$ rRNA groupings were used as the basis for comparison with phylogenetic trees derived from our analysis of LuxI and LuxR family members.

\section{Phylogenetic analysis of the Luxl and LuxR families}

Neighbour-joining trees generated from the aligned LuxI and LuxR sequences indicated that the majority of proteins in each family clustered within the same groups defined by $16 \mathrm{~S}$ rRNA sequences (Figs $2 \& 3$ ). Although individual exceptions to this rule were observed, the overall congruity between the quorum sensing and the rRNA trees is consistent with an ancient origin for quorum sensing proteins within the Proteobacteria.

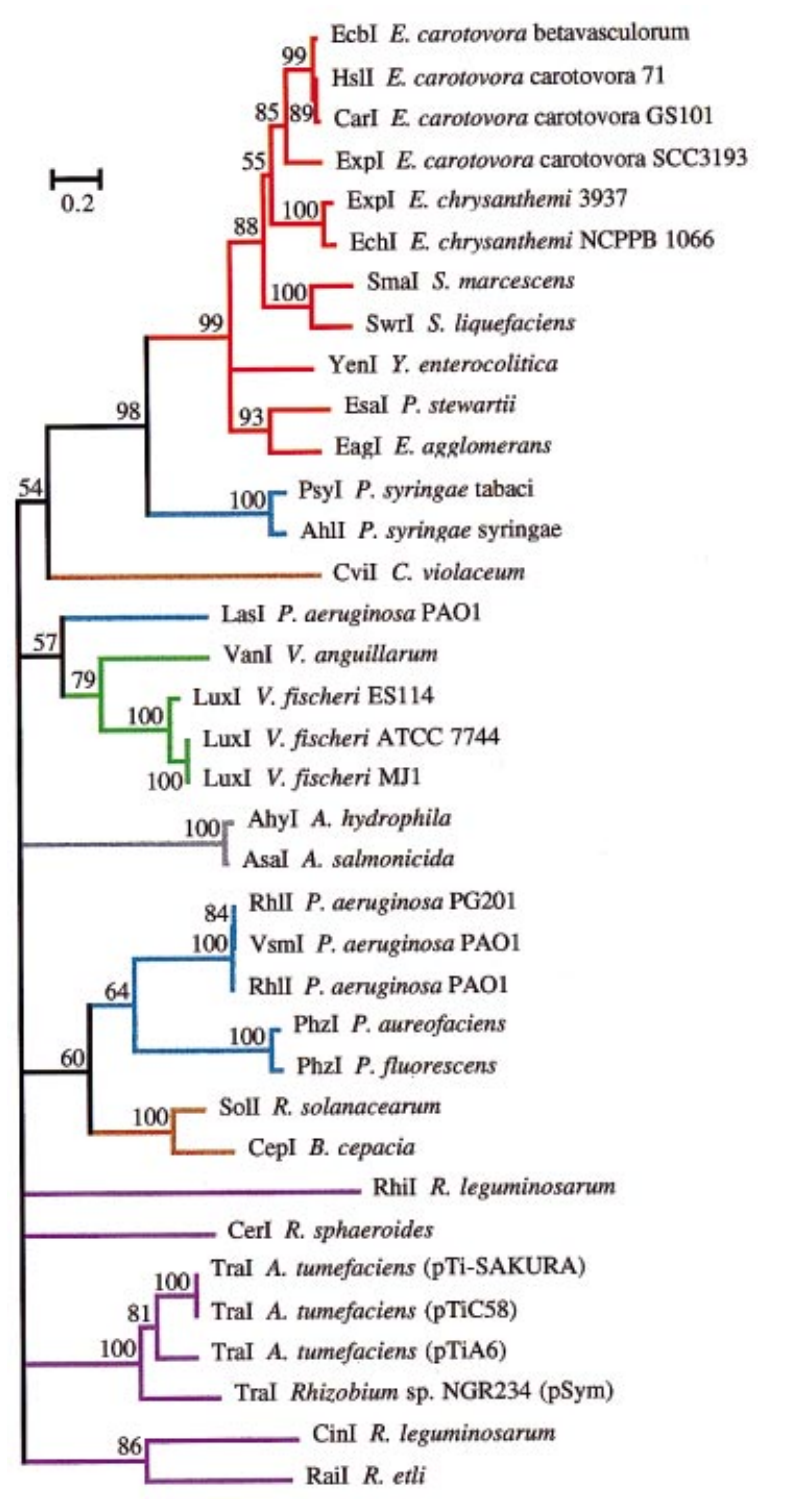

Fig. 2. Neighbour-joining tree of Luxl family members. Scale bar indicates the mean number of substitutions per site. Bootstrap values as the percentage of 500 replicates are shown for each branch with values of $50 \%$ or greater. Branches with less than $50 \%$ bootstrap support are shown collapsed. Colours indicate species belonging to the $\alpha$-Proteobacteria (purple), $\beta$ Proteobacteria (brown), pseudomonads (blue), aeromonads (grey), vibrios (green) and enterobacteria (red).

Similar results were obtained with maximum-parsimony trees. Trees generated by either method using alignments based on previously published comparisons of a more limited number of sequences (Fuqua \& Eberhard, 1999; Stevens \& Greenberg, 1999) were indistinguishable from those produced using our own alignments.

Unfortunately, the amino acid sequences of individual LuxI homologues did not resolve deeper relationships among taxa in this family, as the previously identified groups simply joined with a few unaffiliated homologues 


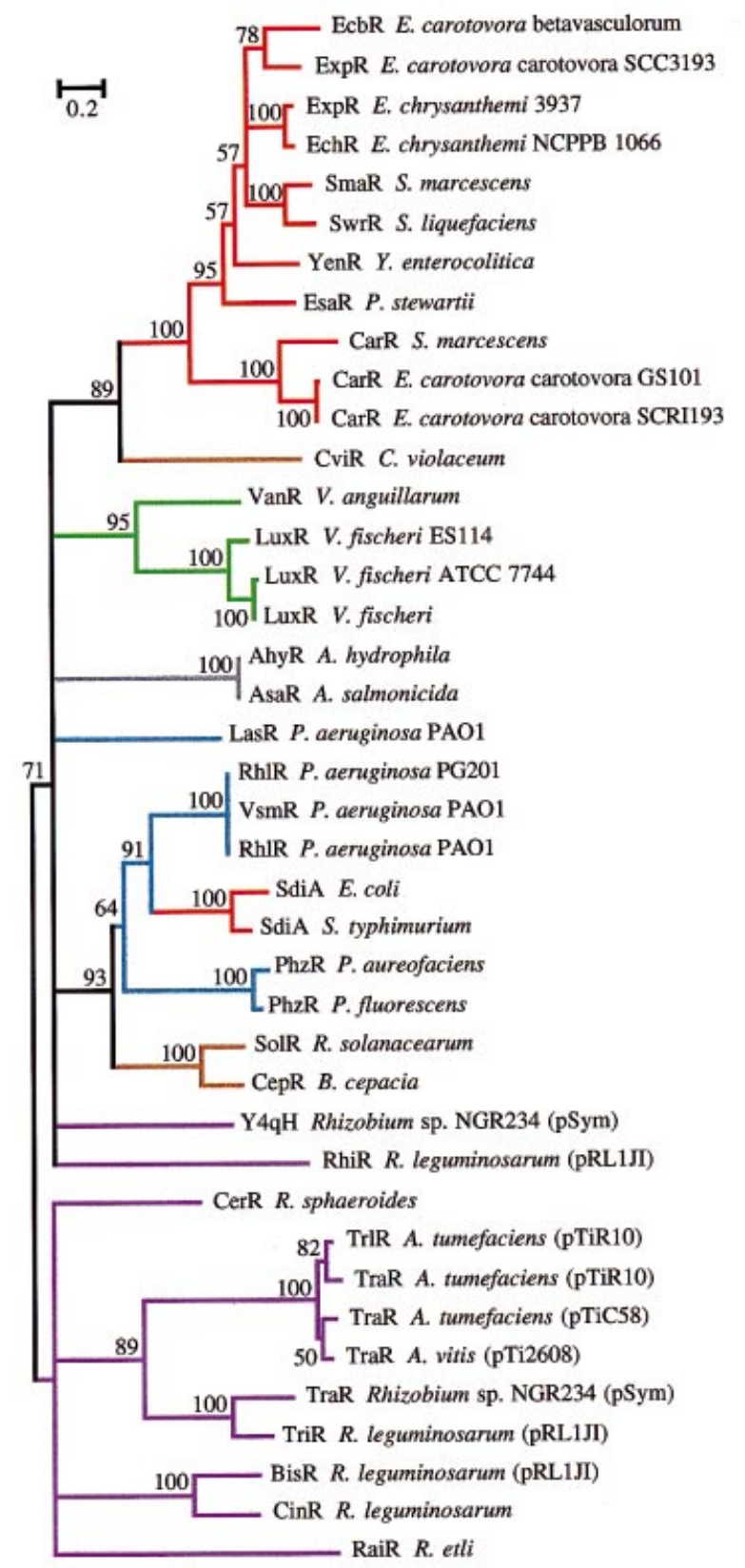

Fig. 3. Neighbour-joining tree of LuxR family members. Scale bar, bootstrap values and colours are the same as for Fig. 2 .

into a single polytomy (Fig. 2). The LuxR family tree showed a similar pattern: in this case, a basal branch separated most of the $\alpha$-proteobacterial sequences from the remaining groups, but these were once again united as a large polytomy (Fig. 3). This basal separation of the $\alpha$-Proteobacteria sequences had relatively weak $(71 \%)$ bootstrap support in the neighbour-joining trees and was absent from the maximum-parsimony trees, which more closely resembled the single polytomy of the LuxI tree.

In an attempt to resolve these uncertain deeper branches further, we decided to consider the combined sequences

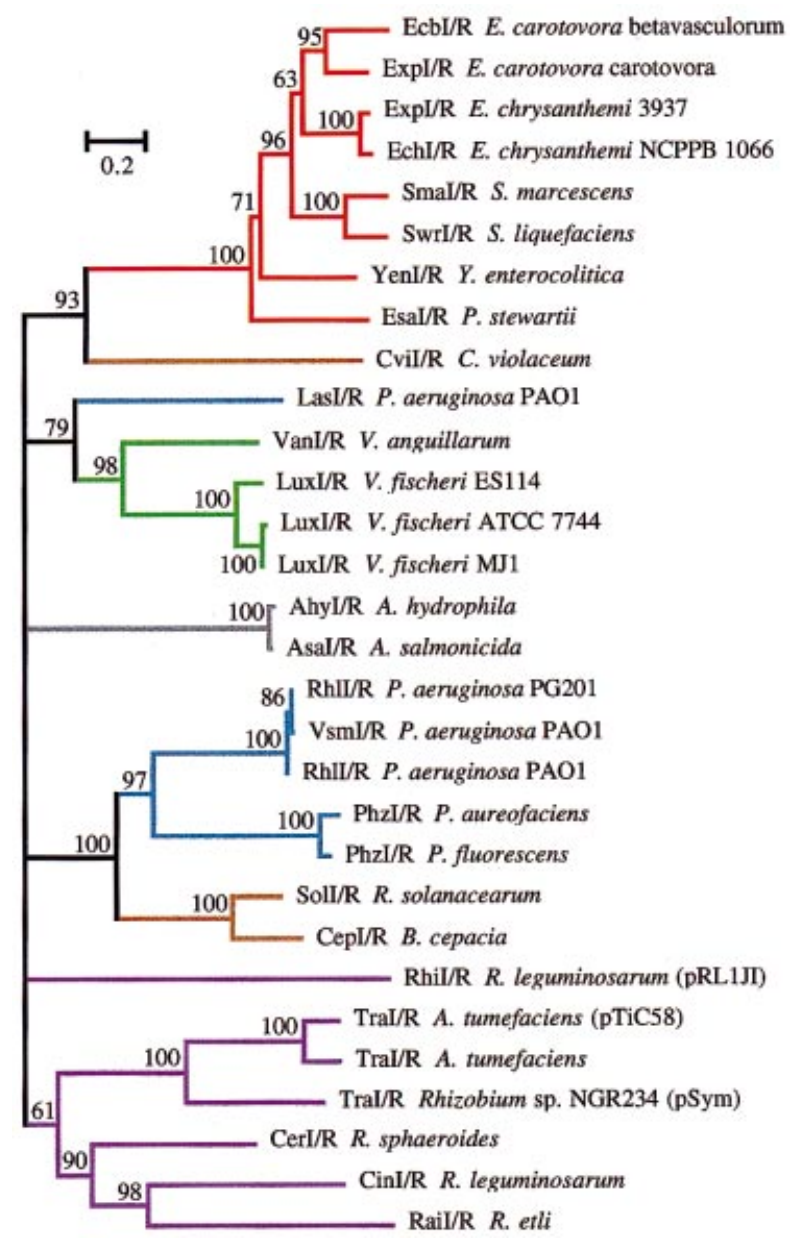

Fig. 4. Neighbour-joining tree of combined LuxI/LuxR sequences from functionally linked ('cognate') gene products. Scale bar, bootstrap values and colours are the same as for Fig. 2.

of 'cognate' pairs of LuxI and LuxR homologues. Cognate pairs are defined as those that are functionally linked, with the LuxI homologue producing the specific acyl homoserine lactone required to activate the corresponding LuxR protein. In most cases, cognate luxI and $\operatorname{luxR}$ gene homologues are located adjacently to each other, with ORFs oriented tandemly, divergently or convergently (Table 1). In addition, the TraI and TraR proteins of Agrobacterium tumefaciens and the Rhil and RhiR proteins of Rhizobium leguminosarum are known to function as cognate pairs, despite the fact that their genes are not adjacently positioned. Because functionally related gene pairs have the potential to coevolve, we could repeat our analysis using the combined sequences of these cognate LuxI and LuxR homologues as single taxa.

The resulting phylogenetic tree from the combined LuxI and LuxR sequences was more robust in terms of bootstrap support than those generated from LuxI or LuxR sequences alone, but was otherwise the same as the previous trees (Fig. 4). The fact that this 'combined' 
tree was consistent with the individual LuxI and LuxR trees supported the hypothesis that cognate $\operatorname{luxI} / \operatorname{lux} R$ gene pairs may have become physically and/or functionally linked fairly early in their history and subsequently coevolved as unified regulatory cassettes. The relative orientation of the ORFs, however, was not a reliable indicator of phylogenetic relationship. As an example, the divergently oriented homologues of Vibrio fischeri grouped most closely with the convergently oriented homologues of Vibrio anguillarum, while both sets of Vibrio sequences clustered with the tandemly oriented Pseudomonas aeruginosa LasI/LasR sequences (Fig. 4, Table 1).

In a final attempt to resolve deeper relationships among the different groups of quorum sensing regulators, we performed an outgroup analysis on the LuxR family tree. LuxR family members are part of a larger superfamily of regulatory proteins that share similarity only in their C-terminal DNA binding domains (Henikoff et al., 1990; Slock et al., 1990). Three members of this superfamily, FixJ from Sinorhizobium meliloti, EpsR from Ralstonia solanacearum and MalT from Escherichia coli, were therefore used as representative outgroups from the $\alpha$-, $\beta$ - and $\gamma$-Proteobacteria, respectively. Unfortunately, these outgroup sequences were so different from the quorum sensing family sequences that the resulting alignments were too weak to provide any useful information in terms of basal associations with the LuxR homologues. As a result, we were unable to establish which of the quorum sensing homologues appeared to be associated most closely with the outgroup sequences.

The observed groupings of the Chromobacterium violaceum Cvil/CviR sequences with the enterobacterial cluster and of the Pseudomonas aeruginosa LasI/LasR sequences with the Vibrio cluster (Fig. 4) represented notable exceptions to the overall agreement between the $16 S$ rRNA gene tree and the combined LuxI/LuxR tree. The validity of these anomalous groupings was tested by placing each pair of sequences into the appropriate host group as identified by $16 \mathrm{~S}$ rRNA analysis and subsequently determining the statistical significance of the resulting differences in the two trees (Templeton, 1983; Kishino \& Hasegawa, 1989).

When the combined CviI/CviR sequences were moved to join the base of the $\beta$-proteobacterial cluster (Fig. 4), the resulting tree was significantly longer than the original $(P=0.0007$ and 0.0012 for the KishinoHasegawa and Templeton tests, respectively). The same result was true of CviR in the LuxR tree alone $(P=$ 0.0002 and 0.0005$)$, but not of CviI in the LuxI tree $(P=$ 0.0513 and 0.0744). These results suggest that the Chromobacterium CviR is more closely related to the enterobacterial LuxR homologues than it is to those of the other $\beta$-Proteobacteria. As the association of CviI with the enterobacterial LuxI sequences was not supported statistically, its proper affiliation remains open to question. By contrast, moving the combined LasI/LasR sequences of Pseudomonas aeruginosa into the pseudo- monad cluster also resulted in a significantly longer tree $(P=0.0137$ and 0.0139$)$, but this difference primarily reflected the contribution of LasI rather than LasR. Moving LasR alone did not produce a significantly longer tree $(P=0.2971$ and $0 \cdot 2985)$, while moving LasI alone did result in a significant difference $(P=0.0024$ and 0.0027). These are intriguing results, given our previous finding concerning the possible coevolution of functionally linked LuxI/LuxR pairs. Apparently CviI/ CviR and LasI/LasR are unusual pairs of cognate quorum sensing homologues in that one member of each pair appears to be affiliated with a specific group of sequences, while the other does not.

\section{Horizontal transfer of individual Luxl and LuxR homologues}

Many other differences between the rRNA and quorum sensing trees were observed among the unlinked homologues found within the separate LuxI and LuxR trees. For example, the LuxI homologues of two pathovars of Pseudomonas syringae grouped strongly $(98 \%$ bootstrap support) at the base of the enterobacterial cluster, well apart from other Psendomonas LuxI homologues (Fig. 2). The appearance of the Pseudomonas syringae sequences within this cluster suggests that this species may have acquired its quorum sensing regulators by means of horizontal gene transfer. Moving these sequences to the base of the pseudomonad cluster resulted in a significantly longer tree $(P=0.005$ and $0 \cdot 006)$, indicating that they properly belong with the enterobacterial group.

Similarly, the unlinked LuxR homologues, both named SdiA, of Escherichia coli and Salmonella typhimurium did not group with other enterobacterial homologues. Instead, they appeared most closely related to the Pseudomonas aeruginosa RhlR sequences (Fig. 3), suggesting the horizontal transfer of a pseudomonad homologue to one or both of these enterobacterial species. Moving the SdiA proteins to the base of the enterobacterial cluster created a significantly longer topology $(P=0 \cdot 0018$ and $0 \cdot 0019)$. The SdiA proteins are physiologically unusual in that no specific autoinducer is known to be involved in their activation, although it has been proposed that they might respond to autoinducers produced by other bacteria (Ahmer et al., 1998). Both Escherichia coli and Salmonella typhimurium have been shown to produce extracellular signals (Surette \& Bassler, 1998), but these compounds do not appear to be acylated homoserine lactones (Surette et al., 1999), nor is there any evidence of a functional link between them and the SdiA proteins (Surette \& Bassler, 1999).

An alternative interpretation of these results is that differences between the quorum sensing and the rRNA trees might reflect gene duplication events followed by the subsequent loss of certain duplicated genes over time. The enterobacterial-like LuxI homologues of Pseudomonas syringae might therefore represent genes that were lost or remain unidentified in the majority of 
pseudomonads. While this argument cannot be ruled out entirely, it does not satisfactorily explain many of the results from this analysis. For example, the complete genome of Escherichia coli (Blattner et al., 1997) contains no LuxI homologues and only the single LuxR homologue SdiA, which resembles a pseudomonad rather than an enterobacterial sequence (Fig. 3). To account for this result by gene duplication, Escherichia coli would have to have lost the LuxI and LuxR homologues retained by almost all other enterobacteria, while retaining instead the pseudomonad-like SdiA, which has yet to be found in the other enterobacterial taxa. In addition, there are several examples (described below) in which multiple quorum sensing regulators are found within a single bacterial species, yet most of these homologues cluster within the same rRNA group that defines the host. As a result, even in situations where we know that multiple homologues exist, those examples do not support the argument that early gene duplication adequately explains the appearance of a pseudomonadlike homologue within an enterobacterial host. We therefore propose that horizontal gene transfer is the best explanation for these instances of observed nonagreement between the quorum sensing regulator and the rRNA trees.

\section{Sequence variability within species}

Surprisingly, the variation observed among quorum sensing homologues from different laboratory strains of the same bacterial species was sometimes greater than the variation observed between closely related species. For example, the LuxI and LuxR proteins of Vibrio fischeri ES114 are distinct from those of strains MJ1 and ATCC 7744 (Fig. 4), while the LuxI homologue ExpI of Erwinia carotovora SCC3193 differs from the synonymous HslI and CarI sequences of strains 71 and GS101 (Fig. 2). By comparison, the quorum sensing proteins found in Aeromonas hydrophila and Aeromonas salmonicida are more closely related to each other, as are those of Pseudomonas fluorescens and Pseudomonas aureofaciens (Fig. 4). This disparity of greater intraspecific than interspecific variability may reflect either recent horizontal transfer events or simply the recent speciation of these closely related taxa. In either case, it is curious that such extreme sequence diversity should exist within regulatory proteins shared by different strains of a single species.

\section{Evolution of multiple homologues within a species}

Six species of bacteria included in this study, Agrobacterium tumefaciens, Erwinia carotovora, Pseudomonas aeruginosa, Rhizobium sp. NGR234, Rhizobium leguminosarum and Serratia marcescens, contain more than one LuxI and/or LuxR family member (Table 1 ). If these multiple homologues arose endogenously by means of recent gene duplication, one would expect them to group most closely with each other in this analysis. Among 13 such possible pairings, however, only two of them - TraR and TrlR of Agrobacterium tumefaciens, and CinR and BisR of Rhizobium leguminosarum - appeared as closest relatives (Fig. 3). This suggests that in most cases the multiple quorum sensing genes present within a single species were each acquired independently of the other.

This result has important implications for the evolution of hierarchical signalling cascades in general. As an example, Rhizobium leguminosarum contains a pair of linked homologues (cinI and cinR) on its chromosome as well as the $\operatorname{luxI}$ homologue rhil and three additional unlinked $\operatorname{lux} R$ homologues (bisR, rhiR and triR) on the Sym plasmid pRL1JI (Table 1). According to our analysis, each of the LuxI and LuxR family members present on this Sym plasmid appears to have been acquired from a separate source (Figs $3 \& 4$ ), with only BisR suggesting a possible endogenous origin from the chromosomal CinR (or vice versa). Given this mosaic pattern of inheritance, it will be especially intriguing to understand how the functions of these different homologues are integrated into a unified global regulatory system. The sheer abundance of these diverse regulators within this species may provide continuing opportunities for innovation in the specific role each plays within this global hierarchy.

Similarly, the two LuxR family members CarR and ExpR of Erwinia carotovora subsp. carotovora (Table 1) allow the independent activation of different sets of genes in response to a single autoinducer signal (McGowan et al., 1995; Pirhonen et al., 1993). Of these two homologues, CarR groups at the base of the enterobacterial cluster with a homologue of the same name found in Serratia marcescens, while ExpR clusters with the other Erwinia sequences (Fig. 4). The same pattern is seen with the two Serratia marcescens homologues CarR and SmaR, which group separately with the Erwinia carotovora CarR and the Serratia liquefaciens SwrR, respectively (Fig. 4). These results again suggest that each of the two LuxR homologues found in these species were acquired independently of each other. In the case of the two CarR proteins, these data further imply that horizontal gene transfer may have occurred between Erwinia and Serratia. Both of the CarR proteins activate carbapenem antibiotic synthesis in their respective hosts (Cox et al., 1998; Holden et al., 1998; Thomson et al., 2000). This conservation of function may result from the fact that each homologue is genetically linked to a carbapenem biosynthesis gene cluster (Cox et al., 1998; Holden et al., 1998; Thomson et al., 2000), thus allowing the inheritance of biosynthetic and regulatory functions as a single cassette.

Finally, the independent acquisition of multiple homologues is also evident in Pseudomonas aeruginosa. As previously noted, the LasI and LasR proteins of Pseudomonas aeruginosa showed no affiliation with any of the other pseudomonad homologues, including RhlI and RhlR from the same species (Fig. 4). This suggests that the lasI and lasR genes were acquired independently of rhll and rhlR. This is an especially significant result given that LasI and LasR serve as dominant regulators of 
rhll and rhlR gene expression in Pseudomonas aeruginosa (Latifi et al., 1996; Pesci et al., 1997).

Collectively, these results provide additional support for the potential importance of horizontal gene transfer in the evolution of bacteria (Lawrence, 1997; Lawrence \& Ochman, 1998). The horizontal transfer of LuxI and LuxR family members is especially noteworthy, however, because these represent regulatory rather than structural genes, and it has been proposed that regulatory functions are not routinely acquired by means of horizontal transfer (Jain et al., 1999). Our results therefore suggest that bacteria not only can gain new functional capabilities through horizontal gene transfer, but that they can gain new regulatory circuits and/or signalling abilities as well. As a specific example, Escherichia coli and Salmonella typhimurium may have the ability to respond to acyl homoserine lactone autoinducers generated by other organisms (Ahmer et al., 1998; Sitnikov et al., 1996), primarily because the ability to process that information through a LuxR homologue was itself acquired from other organisms. The functional organization of the Pseudomonas aeruginosa LasI/LasR and RhlI/RhlR quorum sensing systems provides a further example of the potential for acquired regulatory functions to be incorporated within existing regulatory pathways. These data therefore suggest that integrated, hierarchical signalling cascades can potentially arise through the sequential addition of independently acquired regulatory genes.

\section{ACKNOWLEDGEMENTS}

We thank J. A. Downie, M. Givskov, S. Lewenza, P. Sokol, S. Winans, M. K. Winson and J. Zhu for sharing sequence data in advance of publication. We also thank A. K. Chatterjee, C. Fuqua, P. D. Shaw and P. Williams for helpful information about other unpublished sequences. This work was supported by NSF Grant MCB-9600766 to K.M.G. and USDA Grant 9502133 to J.R.G.

\section{REFERENCES}

Ahmer, B. M., van Reeuwijk, J., Timmers, C. D., Valentine, P. J. \& Heffron, F. (1998). Salmonella typhimurium encodes an SdiA homolog, a putative quorum sensor of the LuxR family, that regulates genes on the virulence plasmid. J Bacteriol 180, 1185-1193.

Blattner, F. R., Plunkett, G., III, Bloch, C. A. \& 14 other authors (1997). The complete genome sequence of Escherichia coli K12. Science 277, 1453-1474.

Cox, A. R. J., Thompson, N. R., Bycroft, B., Stewart, G. S. A. B., Williams, P. \& Salmond, G. P. C. (1998). A pheromone-independent CarR protein controls carbapenem antibiotic synthesis in the opportunistic human pathogen Serratia marcescens. Microbiology 144, 201-209.

Fuqua, C. \& Eberhard, A. (1999). Signal generation in autoinduction systems: synthesis of acylated homoserine lactones by LuxI-type proteins. In Cell-Cell Signaling in Bacteria, pp. 211-230. Edited by G. M. Dunny \& S. C. Winans. Washington, DC: American Society for Microbiology.

Greenberg, E. P. (2000). Acyl-homoserine lactone quorum sensing in bacteria. J Microbiol 38, 117-121.
Henikoff, S., Wallace, J. C. \& Brown, J. P. (1990). Finding protein similarities with nucleotide sequence databases. Methods Enzymol 183, 111-132.

Higgins, D. G. \& Sharp, P. M. (1989). Fast and sensitive multiple sequence alignments on a microcomputer. CABIOS 5, 151-153.

Holden, M. T. G., McGowan, S. J., Bycroft, B. W., Stewart, G. S. A. B., Williams, P. \& Salmond, G. P. C. (1998). Cryptic carbapenem antibiotic production genes are widespread in Erwinia carotovora: facile trans activation by the carR transcriptional regulator. Microbiology 144, 1495-1508.

Jain, R., Rivera, M. C. \& Lake, J. A. (1999). Horizontal gene transfer among genomes: the complexity hypothesis. Proc Natl Acad Sci U S A 96, 3801-3806.

Kishino, H. \& Hasegawa, M. (1989). Evaluation of the maximum likelihood estimate of the evolutionary tree topologies from DNA sequence data, and the branching order in Homonoidea. J Mol Evol 29, 170-179.

Kumar, S., Tamura, K. \& Nei, M. (1994). MEGA: molecular evolutionary genetics analysis software for microcomputers. Comput Applic Biosci 10, 189-191.

Latifi, A., Foglino, M., Tanaka, K., Williams, P. \& Lazdunski, A. (1996). A hierarchical quorum-sensing cascade in Pseudomonas aeruginosa links the transcriptional activators LasR and RhlR (VsmR) to expression of the stationary-phase sigma factor RpoS. Mol Microbiol 21, 1137-1146.

Lawrence, J. G. (1997). Selfish operons and speciation by gene transfer. Trends Microbiol 5, 355-359.

Lawrence, J. G. \& Ochman, H. (1998). Molecular archaeology of the Escherichia coli genome. Proc Natl Acad Sci USA 95, 9413-9417.

McGowan, S., Sebaihia, M., Jones, S. \& 7 other authors (1995). Carbapenem antibiotic production in Erwinia carotovora is regulated by CarR, a homologue of the LuxR transcriptional activator. Microbiology 141, 541-550.

Nicholas, K. B. \& Nicholas, H. B., Jr (1997). GeneDoc: a Tool for Editing and Annotating Multiple Sequence Alignments, http:// www.psc.edu/biomed/genedoc. Pittsburgh, PA: Pittsburgh Supercomputing Center.

Oger, P., Kim, K.-S., Sackett, R. L., Piper, K. R. \& Farrand, S. K. (1998). Octopine-type $\mathrm{Ti}$ plasmids code for a mannopineinducible dominant-negative allele of $t r a R$, the quorum-sensing activator that regulates $\mathrm{Ti}$ plasmid conjugal transfer. Mol Microbiol 27, 277-288.

Parsek, M. R. \& Greenberg, E. P. (2000). Acyl-homoserine lactone quorum sensing in Gram-negative bacteria: a signaling mechanism involved in associations with higher organisms. Proc Natl Acad Sci US A 97, 8789-8793.

Pesci, E. C., Pearson, J. P., Seed, P. C. \& Iglewski, B. H. (1997). Regulation of las and rhl quorum sensing in Pseudomonas aeruginosa. J Bacteriol 179, 3127-3132.

Pirhonen, M., Flego, D., Heikinheimo, R. \& Palva, E. T. (1993). A small diffusible signal molecule is responsible for the global control of virulence and exoenzyme production in the plant pathogen Erwinia carotovora. EMBO J 12, 2467-2476.

Rodelas, B., Lithgow, J. K., Wisniewski-Dye, F., Hardman, A., Wilkinson, A., Economou, A., Williams, P. \& Downie, J. A. (1999). Analysis of quorum-sensing-dependent control of rhizosphereexpressed (rhi) genes in Rhizobium leguminosarum bv. viciae. J Bacteriol 181, 3816-3823.

Shapiro, J. A. (1998). Thinking about bacterial populations as multicellular organisms. Annu Rev Microbiol 52, 81-104.

Sitnikov, D. M., Schineller, J. B. \& Baldwin, T. O. (1996). Control 
of cell division in Escherichia coli: regulation of transcription of fts $Q A$ involves both $r p o S$ and SdiA-mediated autoinduction. Proc Natl Acad Sci US A 93, 336-341.

Slock, J., VanRiet, D., Kolibachuk, D. \& Greenberg, E. P. (1990). Critical regions of the Vibrio fischeri LuxR protein defined by mutational analysis. J Bacteriol 172, 3974-3979.

Stevens, A. M. \& Greenberg, E. P. (1999). Transcriptional activation by LuxR. In Cell-Cell Signaling in Bacteria, pp. 231-242. Edited by G. M. Dunny \& S. C. Winans. Washington, DC: American Society for Microbiology.

Surette, M. G. \& Bassler, B. L. (1998). Quorum sensing in Escherichia coli and Salmonella typhimurium. Proc Natl Acad Sci US A 95, 7046-7050.

Surette, M. G. \& Bassler, B. L. (1999). Regulation of autoinducer production in Salmonella typhimurium. Mol Microbiol 31, 585-595.

Surette, M. G., Miller, M. B. \& Bassler, B. L. (1999). Quorum sensing in Escherichia coli, Salmonella typhimurium, and Vibrio harveyi: a new family of genes responsible for autoinducer production. Proc Natl Acad Sci U S A 96, 1639-1644.

Swift, S., Williams, P. \& Stewart, G. S. A. B. (1999). N-Acyl homoserine lactones and quorum sensing in proteobacteria. In Cell-Cell Signaling in Bacteria, pp. 291-313. Edited by G. M.
Dunny \& S. C. Winans. Washington, DC: American Society for Microbiology.

Swofford, D. L. (2000). PAUP*. Phylogenetic Analysis Using Parsimony ("and Other Methods), Version 4. Sunderland, MA: Sinauer Associates.

Templeton, A. R. (1983). Phylogenetic inference from restriction endonuclease cleavage site maps with particular reference to the evolution of humans and the apes. Evolution 37, 221-244.

Thomson, N. R., Crow, M. A., McGowan, S. J., Cox, A. \& Salmond, G. P. C. (2000). Biosynthesis of carbapenem antibiotic and prodigiosin pigment in Serratia is under quorum sensing control. Mol Microbiol 36, 539-556.

Van de Peer, Y., Caers, A., De Rijk, P. \& De Wachter, R. (1998). Database on the structure of small ribosomal subunit RNA. Nucleic Acids Res 26, 179-182.

Woese, C. R. (1987). Bacterial evolution. Microbiol Rev 51, 221-271.

Zhu, J. \& Winans, S. C. (1998). Activity of the quorum-sensing regulator TraR of Agrobacterium tumefaciens is inhibited by a truncated, dominant-defective TraR-like protein. Mol Microbiol 27, 289-297.

Received 15 February 2001; accepted 1 May 2001. 